\title{
¿Va América Latina hacia la bancarrota?*
}

He sido invitado a pronunciarme sobre un tema altamente controversial y que, por su formulación, atrae y seguirá atrayendo una: gran atención. Sin embargo, dada la generalidad del interés que el mismo suscita y su importancia tanto para los prestamistas como para los prestatarios de todo el mundo, se debe ser doblemente cuidadoso en asegurar que estos comentarios reflejen efectivamente la problemática que tenemos entre manos. En tal sentido, creo sinceramente que los términos de referencia que se me han sugerido son confusos y quizás inapropiados, y a ello me referiré en primer lugar.

\section{LA CONFUSION DE UN TITULO INAIPROPIADO PARA UN TEMA DELICADO}

El título de la exposición sugiere, en primer término, que en la América Latina hay un problema de pagos externos de carácter bomogéneo y grave. Yo también pienso que existe un problema, pero no creo que se trate de algo que pueda ser discutido en términos puramente "regionales" y menos aún que pucda plantearse en términos de una hipotética "bancarrota".

Debe enfatizarse, una vez más, que América Latina es una región sumamente heterogenea y que el planteamiento y la importancia relativa de los problemas cambia considerablemente según el país o los grupos de paises que se examinen.

Es muy claro, por ejemplo, que hay en la región países exporta. dores de petróleo que son acreedores netos de la economía mundial. Hay otros paises también que han alcanzado en los últimos años fuertes posiciones en materia de reservas internacionales, que disipan cualquier preocupación respecto a su capacidad para pagar sus obligaciones pendientes.

Hay, en él otro extremo del espectro, un número de países con obligaciones externas pesadas y que han provocado la preocupación reciente de los medios financieros internacionales. Sin embargo, aun en esos casos, hay algunas economías que por razones vinculaclas a su estructura productiva interna, o por diferentes azares de la coyuntụra mundial, se encuentran en mejor situación que otros para hacer frente a sus obligaciones, en el corto o en el largo plazo.

- Basado en la exposición efcctuada por el autor en la conferencia sobre BdNa Latinonmerigana organizada en Caracas por el Financial Times, de Londres, en octubre de 1977 . 
El lítulo pareciera sugerir además que este problemal alectó básicamente a la América Latina. Debe recordarse que estos países no están solos. Los problemas de pagos se han desparramaclo por todo el mundo en desarrolto y aun han penetrado scriamente en algunas economias industrializadas, como es el caso de Gran Bretaña e Italia. $\mathrm{Y}$, quizás en otro plano, problemas igualmente nuevos han llegado hasta los balances de pagos cle algunos países del área socialista.

El tema no es, pues, sólo y exclusivamente latinoamericano. Se trata, en realidad, de un tema universal, que puede y debe ser analizado a niveles regionales, pero teniendo siempre presente sus cicterminantes de tipo munclial para que el enfoque sea realmente comprensivo.

Es claro, pues, que la discusión no debiera focalizarse en América Latina en su conjunto, sino quizás en aquellas situaciones especiales que crean inquictudes tanto a los propios países afectados por ellas como a los medios financieros internacionales.

No es, por cierto, el propósito de está exposición entrar al análisis de tales casos. No es ésta la ocasión ni el lugar para hacerlo, pero no podría dejar de señalar el hecho. Voy a limitarme a formular algunas reflexiones generales en torno a estos problemas para que cle cllas se infieran implicaciones de políticas susceptibles de aplicarse a casos concretos.

Desco, en segundo lugar, referirme al término "bancarrota", que figura en el título de esta conferencia. En verdad, pienso que él está muy lejos de clescribir en forma precisa la situación a que se refiere.

A primera vista, la incapaciliad de un país para hacer frente a sus obligaciones pareciera sugerir un caso similar al de un individuo o una empresa en situación de insolvencia. Sin embargo, como es obvio, esta analogía no puede llevarse muy lejos.

En los casos de las personas o las sociedades, una situación de bancarrota implica la liquidación compulsiva de sus activos por la masa de acreedores y la distribución entre ellos de los recursos resultantes. Además, a menudo, el resultado final de este proceso genera sólo una recuperación parcial de las sumas prestadas.

En cambio, por definición, los países no pueden ir a la bancarrota. Una nación sobcrana no sc ve arrastracla a las cortes sin su consentimiento, ni puecle ver liquidados compulsivamente sus activos nacionales. Por lo tanto, ningún país puede ser liquidado o aliviaclo de sus cargas u obligaciones financieras a partir de declaraciones unilaterales de falencia.

Este hecho es, por lo demás, bien conocido por las instituciones internacionales y la banca en general. Una de las razones por las cuales los préstamos a los paises en desarrollo se han vuelto populatres en los últimos tiempos, es por el hecho de que los bancos reconocen que, a difcrencia de los individuos o de las empresas, los paí- 
ses permanecen en la comunidad internacional, aun después de severas crisis linancieras, y deben en frentar y' enfrentan sus compromisos internacionales.

Ello explica el porqué en la experiencia histórica el repudio de la deuda scat una excepción aislada $y$, en cambio, el refinamiento, la reestructuración de la deuda y la prórroga de los plazos sean la regia general para hacer frente a los problemas de pagos circunstanciales por los que atraviesan los paises.

En resumen, en raxón de los argumentos presentados, mi respuesta a la interrogante que figura ell el titulo de esta conferencia es un enfático $y$ rotundo no.

Pero nuestra preocupación no podria limitarse solamente a poner en claro las inconsistencias semánticas del tema. Hay un problema de balince de pagos en el munclo y lo hay también para algunos países de América Latina. ¿Cuáles son sus raícesi ¿Cómo encontrar sus soluciones? Veamos, en primer lugar, los hechos tal como se presentan, atn a riesgo de incurrir en generalizaciones de las que nos hemos prevenido precedentemente.

\section{EL ANALISIS DE LAS CIFRAS}

La relevancia y' preocupación sobre el problema acumulado de la deuda externa de los paises en clesturollo no exportadores de petró. leo surgen ciel análisis frio de las cifras.

DEUDA DESEMBOLSADA DE LOS PAISES EN DESARROLLO NO EXPORTADORES DE PETROLEO

(Miles de millonus de dólares)

\begin{tabular}{lcccc}
\hline & 1973 & 1974 & 1975 & 1976 \\
\hline Deuda garantizatia & 65 & 80 & 98 & 117 \\
Deuda no ganantizada & 23 & 29 & 37 & 49 \\
\hline TordL & 88 & 109 & 135 & 166 \\
\hline
\end{tabular}

Detrás de estas cifras se encuentra, fundanentalmente, el déficit en cuenta corriente del balance de pagos de estos mismos países.

DEFICIT CORRIENTE DEL BALANCE DE PAGOS DE LOS PALSESEN:DESARROLLO NO EXPORTADORES DE PETROLEO.

(Miles de millones de dolares)

\begin{tabular}{rrrrr}
1973 & 1974 & 1975 & 1976 & 1977 \\
-11 & -30 & -38 & -26 & -23 \\
\hline
\end{tabular}


Para hacer frente a estos déficit, los paises no exportadores cle petrólco debieron recurrir al endeudamiento externo, el que para e-te conjunto de países fue provisto en un $50 \%$ por el sector financiero privado:

Cono se ve, las cilras muestran un aumento preocupante del cndëudamiento global de los países en vias de clesarrollo no exportadörés de petróleo. Pero ellas también insinúan una nacla despreciable recuperación durante el año 1976. Las medidas de ạ̣uste interno (restriciones a las importaciones, aumento dic las erportaciones, ajuste en el nivel de actividadi y algunos factores favorables de origen externo (como la evolución cle los precios de algunas materias primas) han provocado la sana reacción que muestran las cifras sobre el cléficit de ia cuenta corriente del balance de pagos.

Sin embargo, a pesar de esta recuperación, estos países deb:eron continuar recumiendo al endeudamiento externo para lalcer frente a sus déficit y recomponer sus reservas, Ias que -bueno es puntualizarlo- ascendieron a 42 millones de dólares a fines del irĩo pasado, la cifra más alta alcanzada por este grupo de paises en todal su lístoria.

¿Que ha pasado, a sü vez, en América Latina? Las cifras respectivas son las siguientes:

DEUDA DESEMBOLSADA DE LOS PAISES NO EXPORTADORES DE PETROLEO DE AMERICA LATINA

(Miles de miliones de dólares)

\begin{tabular}{lcccc}
\hline & 1973 & 1977 & 1975 & 1976 \\
\hline Deuda gurantizada & 24 & 31 & 39 & 49 \\
Detada no garantizada & 14 & 21 & 26 & 35 \\
\hline TotaL & 38 & 52 & 64 & 84 \\
\hline
\end{tabular}

DEFICIT EN CUENTA CORRIENTE DEL BALANCE DE PAGOS DE LOS PAISES NO EXPORTADORES DE PETROLEO DE AMERICA LATINA

(Milies de millones de dólares)

\begin{tabular}{|c|c|c|c|c|}
\hline-1973 & $1974^{\circ}$ & 1975 & 1976 & .1977 \\
\hline-1.2 & -13.1 & -16.2 & -11.2 & -8 \\
\hline
\end{tabular}

Por:otra parte, durante estos años ha ocurrido un cambio significativo en la estructura de la deucla global latinoamericana en lo que átãũe 2 su fuente de financiamiento. 
ESTRUCTURA DEL FINANCKAMIENTO DE LA DEUDA GLOBAL LATINOANERICANA

$\because \quad$.. . (Porcentajes)

\begin{tabular}{lllll}
\hline & 1973 & 1974 & 1975 & 1976 \\
\cline { 2 - 5 } Deuda global & 100 & 100 & 100 & 100 \\
\hline & & & & \\
Privada & 73 & 78 & 78 & 81 \\
Oricial & 27 & 29 & 22 & 19 \\
\hline
\end{tabular}

En efecto, mientras que en los años 60 el grueso cle la deuda latinoamericana provenía de fuentes públicas, en los últimos años ha sido el financiamiento privado el que ha suministrado la proporción fundamental de los fondos.

Como resultado de los cambios señalados, la relación entre la cleuda garantizada y las exportaciones da las siguientes proporciones:

RELACION DEUDA GARANTIZADA A EXPORTACIONES

\begin{tabular}{lrrrr}
\hline & 1973 & 1974 & 1975 & 1976 \\
\cline { 2 - 5 } & & & & \\
1. Deuda garantizada & 23.8 & 31.4 & 38.7 & 49.2 \\
2. Exportaciones & 23.9 & 31.4 & 31.7 & 36.1 \\
3. Coeficiente (1): (2) & 1.0 & 1.0 & 1.22 & 1.34 \\
\hline
\end{tabular}

¿Qué conclusiones se desprenden de las cifras precedentes? Aün a despecho del fuerte nivel de agregación de las informaciones presentadas y de la especial situación de un grupo de países dentro del total, pueden derivarse algunas conciusiones principales.

La primera es que la deuda de la región -que se concentra. especialmente en algunos países- ha alcanzado nivelcs sin precedentes en la experiencia histórica. También sin precedentes han sido las cifras alcanzadas por la exportación de bienes y servicios, pero, a pesar de ello, la relación entre deuda y exportaciones ha pasado de 1 a 1.3 en un perioclo de 4 años.

Sin embargo, vistas en términos clinúmicos, las cifras revelan otro hecho de importancia, cual es el mejoramiento en el balance comercial: en efecto, en 1974 se produjo el gran. salto en el déficit comercial de la región, que pasó de 4 mil millones de dólares en 1973 a 13 mil millones de dólares en 1974; como consecuencia de los aumentos en los precios de los energéticos, de las materias primas; $y$ 
de los insumos y equipos importaclos. La situación se agravó durante 1975 , pero a partir de ese año empezó a recuperarse en formal sosteniclat, $y$ asi se espera que durante 1977 el défícil de la cuental corriente no supere a los 8 mil millones de dólares, cifra equivalente a la mitad de la del desequilibrio registrado dos años atrás. Es preciso considerar, además, que las reservas oficiales -que decrecieron en más de 3 mil millones de clólares en 1975 - se incrementaron en 2 mil millones en 1976, y se estima que han sub:to otros 3 mil millones en lo que va corrido de este año.

Por otra parte, y tal como lo destaca en un reciente articulo el Economista Jele del Banco Mundial para la América Latina, señor John Holsen, el aumento del coeficiente del servicio de la deuda, tal como se registra en las cirras convencionales, exatgera la carga real cle la misma al no distinguir entre obligaciones por intereses y obligaciones por amortizaciones del principal. Representando el pago por amortizaciones los dos tercios del futuro scrvicio, hay un margen considerable para el refinancianiento o la renegociación de plazos más espaciaclos.

Además, los créditos financieros con tasas de interés flotante son más fáciles de refinanciar, siempre que se cumpla el servicio de los intereses y que se mantengat un nivel adecuado de credibilidad en la gestión financiera y en las perspectivats económicas cle los países. También es conveniente recordar que en la deuda se contabilizan las transacciones linancieras entre empresas matrices cxtranjeras $y$ subsidiarias locales, las cuales alcanzan una suma no clespreciable, y que constituyen alternativas contables a una verdaclera inversión directa y son mucho más fáciles de refinanciar. Asimismo, Hotsen señala que la existencia de un alto volumen de créditos no vinculaldos a importaciones o proyectos, facilita la administración de los mismos a los prestatarios.

\section{ALGUNAS REFLEXIONES SOBRE LOS HLCHOS RECIENTES}

A esta altura de la presentación, vale la pena hacer algunas reflexiones de orden general, que puedan ayudar a orientar la acción y situar en forma un poco más precisa nuestra posición en relación al problema:

\section{a) A propósito de la magnilud de la deuda.}

Como se ha visto, no puede ignorarse la existencia cle un problema de rápida acumulación de deuda externa en los países en desarrollo no exportadores de petróleo, el cual afectó especialmentc a algunos paises de ingresos intermedios, como son la mayor parte de los latinoamericanos. 
Como sostiene el Fondo Monetario Internacional en el reciente informe de su asamblea anual:

"Es claro que el surgimiento de clesbalances en cuenta corriente entre los paises en desarrollo no exportadores de petróleo descle 1973 a 1975 sobrepasó la marca. Para hacer frente a las presiones de emergencia del periodo, cuando un ciclo adverso se combinó con el aumento de costos de energia y alimentación, muchos de estos paises debieron confiar en el endeudamiento externo, a tasas y costos que no cran sostenibles, ni clescle el punto de vistal de su prop:o interés ni descle el de los atreedores."

Frente a desequilibrios inesperados en sus bulances de pagos, provocados por los cambios adversos en los precios de sus importaciones y exportaciones, los países enfrentaron el dilema de operar una contracción de sus economías que hubiera siclo políticá y socialmente imposible o de asumir un cndeudamiento creciente. En la mayoria cle los casos, ellos se inclinaron por esta última opción.

Con ello resolvieron su problemi inmediato de creciniento a costa de un mayor endeudamiento, pero también mantuvieron un alto nivel de importaciones, contribuyendo así a lacilitar la recuperación cle los países inclustriales.

\section{b) A propósito do la capacidad de reacción de los paises latinoamericanos}

No basta, por cierto, con contabilizar la magnitud de la deuda. Importa, asimismo, destacar la capaciclacl de reacción de los paises para manejar su situación externa.

Con muy escasas excepciones, los países latinoamericanos adoptaron luertes mediclas de ajustc que contribuyeron a reducir la tasa cle crecimiento global de la región en 1975 a prácticamente la mitad del ritmo registrado en la primera mitad del clecenio en curso.

Así, para frenar la expansión de la demanda y moderar por esa via las presiones sobre las importaciones, se aplicaron distintas meclidas de ajuste en el campo fiscal, monetario y cambiario. A lo anterior, se añadieron políticas para contener directamente las importaciones, y con el mismo fin se sacrificó en parte el nivel de inversión.

Pero la reacción mís notable se refiere a la movilización de las exportaciones. Al respecto, no debe pasar inadvertido el hecho de que en los últimos cuatro años el ritmo de crecimiento del volumen físico de las exportaciones latinoamericanas se mantuvo $y$, lo que es más importante, las politicas de apertura externa y de reducción de aranceles no se sacrificaron en razón de la adversa coyuntura internacional. América Latina realizó, asi, una contribución significativa 
para la mantención del nivel de actividad en la economịa muncial y para la recuperación cíclica de los países industriales.

. Sin embargo, es evidente que el ritmo de ajuste intcrno tiene rus límites.

Dentro del deséquilibrio actualmente exisţente en el comercio internacional, doncle existen grupos de paises con grandes superávit y otros con grandes cléficit, el endeudamiento de los países deficitarios es el puente natural y necesario para el sostenimiento del sistema financiero internacional, pero es también el punto de apoyo para dosificar la magnitud de los esfuerzos que deberían realizar ambos grupos de países en períodos como los presentes.

En efecto, en el origen de los actuales desequilibrios financieros internacionales no están sólo los problemas o errores cle politica de los paises deficitarios, sino también los de los paises superavitarios. lis evidente, por tanto, que se podría lograr un ajuste bastante más rápido y menos traumático de la situación presente si los paises tomaran medidas tendientes tanto a reducir los déficit como a moderar los superávit, evitando así que todo el peso del ajuste recaiga, como ha ocurrido hasta ahora, sobre las economías deficitarias.

\section{c) A propósito de la reacción de la comunidad internacional $\therefore$ : :}

En los últimos años ha habido una reacción de la comunidad internacional para hacerse cargo del problema y propiciar mecanismos de ayuda financiera de emergencia a los paises más afectados por la crisis de la economía mundial.

El problema, sin embargo, dista mucho de estar resuelto. Aun las iniciatives emprendidas a nivel mundiat. en el marco de las discusiones en torno a un Nuevo Orden Económico Intemacional se encuentran en estado embrionario, y se está muy lejos de haberse encontrado soluciones.

Pero lo que sí resulta claro es que en ese panorana América Latina, clebido a la especial posición relativa en que se encuentra frente al resto de las economías del Tercer Mundo, y al igual que otros paises de desarrollo intermedio, no encontró en las fuentes públicas de crédito la solución transitoria a sus problemas, sino que clebió recurrir fundamentalmente al endeudamiento de tipo privado. Dicho en otros términos, la comunidad internacional no ha dispuesto de mediclas simécricas que permitan dar una respuesta aclecuada a los problemas especiales de los paises de ingresos medios, y ello explica que éstos hayan tenido que recurrir a las fuentes privadas como su fucnte casi única de financiamiento.

- Mirando hacia los años próximos, el problema de la deuda parece ser.significativo especialmente para algunos países de la región, 
pero - conviene insistir una vez más- las situaciones cambian drásticamente según los casos.

Entre otros factores pertinentes, influyen en esta divers:dad de situaciones la particular coyuntura internacional en cuanto al precio de lạs materias primas, la capacidad de gestión y administración para la captación de recursos externos de origen privado, y la mayor o menor capacidad politica y técnica para poner.en práctica ajustes en las políticas internas.

No obstante, la coyuntura inestable en que se encuentra la economía internacional, la indecisa recuperación cle los grandes centros industriales, y ciertos signos equivocos que emergen a raíz de los rebrotes de políticas proteccionistas en algunos cle ellos, hacen temer que la difícil situación de algunos países se repita en otros.

Es preciso, pucs, prepararse para un período de ajuste tanto internacional como nacional, al que hay que abocarse con criterios claros y' con políticas múltiples y en varios niveles.

Es para ubicarnos en esa línea que nos permitiremos hacer algunas consideraciones sobre posibles politicas, sin entrar, por las razones ya anotadas, en el examen de casos específicos.

\section{LOS DISTINTOS NIYELES DE LAS SOLUCIONES}

Antes de en frascarnos en algunas consideraciones en torno a los distinlos planos en que deberán ubicarse las soluciones at problema, valdría la pena diiucidar una pregunta básica que precede a cualquier otra: ¿Habra llegado la régión al máximo de st capacidad de endoudamiento? ¿Estamos, pues, en presencia de un problema mucho más profundo que el que denuncian las cifras resullantes de una coyuntura especial como la vivida en los últimos años por la comunidad in tspmacional?

A nuestro juicio la respuesta es clara: consideramos que la región dista bastante de haber llegado al limite de su capaciclad potencial de endeudamiento. Para ello nos basamos en algtinas consideració nes relacionadas con la evolución de la cconomía regional en jos ultimos años y con sus perspectivas futuras.

Es conociclo el cxtraordinario dinamisino alcanzado por América Latina en la movilización de sus fuerzas productivas. Ellas permitieron aicanzar niveles de crecimiento económico que superaron largamente las expectativas de la Estrategia Internacional del. Desarrollo, excedicndo el $6 \%$ de promedio en años recientes, y en algunos casos superando luertemente esa cifra.

Mirando hacia adelante no hay ninguna razón para que esa dinámica: no persista. Los recursos inaturales, humanos, financieros y 
tecriológicos de América Latina así lo atestiguan. No menos significativa es I" mayor experiencia y madurez cle las políticas económicas puestas en práctica en la mayoría de los países de la región.

Conjuntamente con esa clinámica interna, se ha producido un proceso de apertura externa igualmente notable. Destacamos ya cómo las exportaciones latinoamericanas crecieron mas cle un $50 \%$ en el perfodo 1973-1976. Pero esa ajertura no ha sido tan sólo comercial, sino que se ha extendiclo al canpo financiero $y$ al del interrelacionamiento entre las economías nacionales $y$ el mundo industrializado a distintos niveles.

Esa apertura permitió a América Latina alcanzar niveles cle comercio que no solamente interesan a la región sino que se han converticlo progresivamente en aportes considerables para la dinámici del sistema económico mundial y, en particular, para el de los grandes centros industriales. En otras palabras, la región cuenta hoy no sólo como "importador a" sino como "exponaldom" y su volumen potencial de demanda y oferta se harai sentir mucho más en el futuro si, como es de esperar, los grandes centros abren sus puertas a las exportaciones latinoamericanas.

En ese contexto -potencial econónico interno, apertura externa $y$ adecuadas políticas para asegurar un manejo pruclente de la deuda- el que nos hace creer en la capacidad cle la región palia absorber dosis mayores de endeudamiento a las alcanzadas en la actualidacl. Ello será particularmente necesario en un continente que necesitará de abundantes recursos financieros para desarrollar en el luturo sus considerables fuerzas productivas.

En ese contexto dinámico, el problema de la deucia acumulada y las restricciones que a mediano plazo ella plantea para algunos paises no debieran oscurecer las perspectivas de más Iargo plazo, vinculadas al alto potencial económico de lit región y a su capacidad para alcanzar ritmos aun más altos de crecimiento que los lograclos en los últimos tiempos.

¿Cuáles son, pues, los niveles actuales en los que debe encuadrarse el problema para hacer frente al periodo de transición en que se encuentran nuestras economias y algunos prises en particular?

a) El primer nivel de las soluciones debe ubicarse al nivel mundial $y$ en particular en las politicas que deben espstrarse de los grandes centros industriales

Ya mencionamos un primer componente de la responsabilidad internacional: el de buscar adecuadas soluciones a los problemas de los países deficitarios, patrocinando ajustes progresivos en que también participen los países superavitalios, que permitan disminuir la pesaḍa carga que aquéllos representan para los primeros. 
Otro componente ha de ser necesilyamente la recuperación de las economías centrales. Todos estamos conscientes de la complejidad que ello involucra. Pero resulta claro que muchos de los problemas del endeudamiento están ligados a la salud económica de los grandes centros $y$, en particular, al mayor nivel de su actividad interna. De éste dlepende, en efecto, en medidla considerable aunque no exclusiva, la fortaleza de los precios de los productos básicos. Y' es preciso recordar que la recuperación de los precios cle algunos productos básicos seguirá siendo la clave fundamental para la solución de los problemas de la balanza do pagos de ciertos países en desarrollo. Por eso, tanto la recuperación de las economías centrales como la bús. queda de soluciones integrales al problema de los precios de los productos básicos, siguen siendo piezats claves en el proceso de búsqueda de soluciones a la crisis de la balanza de pagos de los paises en desarrollo.

Pero no basta con eso. Debemos insistir, una vez más, en lo que significa para las economías periféricas, $y \in n$ particular las de ingresos medios, contar con mercados abiertos a su crecienle vocación exportadora.

En este sentidio, no podemos dlejar de haccr notar y de lamentar Ios claros signos de un creciente proteccionismo que se advierten en las economias centrales. En recientes informes del Fondo Nonetario $y^{\prime}$ de otros organismos internacionales se han analizado estas tendencias especialmente en el campo de las restricciones cuantitativas al comercio exterior, las cuiles afectan en forma clirecta a los países en vias de desarrollo. En ese campo importa reconocer que la región ha abordado con valentít el proceso de apertura siguiendo una línea sostenida de reclucción de sus aranceles. Justo es, en consecuencia, esperar en contrapartida un acceso lacilitado al los mercado inclustriales.

Sin embargo, por clesgracia, no parece ser esa la reacción en algunos centros industriales. El problemia nos prcocupa, por encle, legitimamente. Por qué no se podria esperar un equilibrio de nuestros pagos extcrnos si se nos recorta la posibilidad de expandir nuestras ventas en el exterior. $Y$ al mismo tiempo no debería perderse de vista que, como ya lo expresáramos, exportaciones crecientes de la región tienen como contrapaticlat importaciones también crecientes. $Y$ como es obvio, éstas servirian, en última instancia, para estimular la demanda en los grandes centros inclustriales a yudando con ello a su propiá recuperación.

Otro tanto podria decirse de la apertura de los mercados financieros internacionales. Ha habido en los últimos tiempos facilidades al acceso a los mercados financieros privaclos, especialmente los bancarios. Sin embargo, no ha habido una igual reacción en los 
mercaclos de capitales, en los cuales todavía la participación cle la región es mínima o simbólica. Debe pensarse que en el futuro nuestros paises proseguirán sus esfuerzos de apertura externa, lo cual supondrá su participación creciente en las múltiples formas de captación de capitales existentes. $Y$ para ello deberán arbitrarse las facilidades y medidas adecuadas por parte de los centros exportadores cle capitales.

En definitiva, ajustes a nivel mundial, recuperación de los precios de los productos búsicos, apertura de los mercados extcrnos, mayor acceso a los mercaclos de capitales, constituyen respuestas al problema que nos preocupa. Pero, para alcanzarlas, no basta con la buena disposición de los países periféricos. Se requiere, además, y primordialmente, la cooperación de los países industrializados.

Es de esperar, por ende, que las negociaciones tendientes a establecer un Nuevo Orden Económico Internacional basado en un reconocimiento, hoy mucho más claro que ayer, de la interdependencia recíproca entre los paises y los procesos económicos, aliente la búsqueda de soluciones en todos estos campos y nos prevenga contra una nueva ola de proteccionismo que, en definitiva, no hará más que agravar el problema no sólo de los paises en desarrollo sino cle la comunidad internacional toda,

\section{b) El segundo nivel es en el de las propias politicas económicas financioras nacionales}

Es en cste campo donde han tenido lugar tas mayores reacciones, los mayores ajustes $y$, por endc, los mayores costos tanto sociales como económicos.

Tal como lo destacáramos precedentemente, los países respondicron a los problemas de corto plazo en sus balances de pagos poniendo en marcha muy variados mecanismos internos. La mayoría de ellos tendrá que continuar aplicíndolos en el futuro para hacer frente con criterios pragmáticos y flexibles al período de ajuste que queda por delante.

Esas políticas incluyen, por cicrto, una vigilancia constante de las políticas fiscales, monetarias y cambiarias $y$, en forma particular, del propio comportamiento de la deuda externa. Al respecto, conviene señalar que la crisis reciente ha revelaclo que en la mayoria de los países de la región hay hoy una mayor capacidad para administrar los procesos cle endeudamiento, y que en ellos hay también una conciencia más clara de la necesidad de políticas integradas y coherentes para hacerles frente y para sostencr la capacidad de crédito $y$ de endeudamiento. Esas políticas prudentes y cuidadosas debieran continuarse $y$, desde luego, enmarcarse dentro de las prioridades y objetivos de una politica global de clesarrollo. 
Pero es bueno también anotar otras lecciones que ha clejaclo en alguna forma el reciente periodo critico por el cual atravesaron algunas economias de la región. En efecto, han quedado de manifiesto algunos clesequilibrios en su proceso de desarrollo que obligan a reflexionar sobre las tendencias futuras del crecimiento y sus correctivos.

La vocación exportadora de la región puso cle manifiesto la necesidad de persistir en una línea de apertura externa y de Comento dinámico y vigoroso cle sus exportaciones, tanto tracliciontles como no tradicionales.

Pero también nos alertó sobre lak nocesidad de no clescuidar los mercados internos, y de aprovechar el gran potencial que tiene la región (y algunos paises en particular) para avalnzar en el campo de la sustitución de importaciones a nivel regional, y con ello superar las insuficiencias que se atlvierten en ciertas ramas o sectores industriales que han quedaclo atrasados.

Algunos paises han reconocido este hecho y' se han lanzado a programas especiales tendientes a llenar la brecha en el desarrollo de algunos sectores claves como son ciertas ramas productoras de bienes de capital y de bienes intermedios que habian permanecido retrasados.

Todos estos ajustes en el modelo de crecimiento exigen una visión de largo plazo de las economías regionales y un equilibrio más adecuado entre los esfuerzos externos e internos. Estamos ciertos que a largo plazo ellos habrain de dar sus frutos y' con ello permitirán una inserción más estable y equilibrada de nuestras economías en el mundo exterior.

Asi, pues, como consecuencia de la reciente crisis financiera, y especialmente como mecanismo de clelensa frente al la inestabilidad en la situación económica mundial, ha surgido una revalorización de los mercados internos $y^{\prime}$ del papel potencial de la cooperación y de la integración regional. Dentro de ese orden de ideas, no debiera clescartarse la importancia de acciones conjuntas entre los propios países de da región.

Oportunamente la oEpat sugirió que se creara un mecanismo de cooperación financiera regional para hacer frente a las situaciones críticas de emergencia de la economia regional. Seguimos creyendo que ideas cle esa naturaleza $y$, en general, una mayor cooperación financiera entre los paises de la región puede ser un ingrediente adicional de importancia en el enfoque de estos problemas. Creaciones de organizaciones bancarias (como el recientemente acordaclo Banco Latinoamericano de Exportaciones) son igualmente respuestals que van sumanclo esfuerzos a la solución conjunta del problema. 
c) Por iltimo, corresponde igualmente comprometer en la búsqueda de solucionss al propio sistema financicro internacional

Ya hemos visto la importancia que los mecanismos financieros privados han teniclo para solucionar en el corto plazo los cléficit corrientes en la balanza de pagos cle la región. Esa importancia habrá de persistir en el futuro inmediato.

Es claro que el proceso de ajuste a nivel internacional habrá de ser lento y difícil. En primer término, porque, tanto en los países con superávit como deficitarios, los ajustes requieren medidas que at menudo son políticamente impopuiares y difíciles de implantar internamente. Aclemis, dada nuestra interclependencia global, un ajuste exitoso requiere siempre de un graclo de coordinación en las decisiones de politicis a nivel internacional que aún no se ha alcanzado en un munclo acostumbraclo al imperio de la soberanía en la adopción de las políticas nacionales.

$\mathrm{Si}$, por lo tanto, no es previsible un ajuste rápido a corto plazo, es entonces de fundamental importancia que se continúe disponiendo de adecuados mecanismos para la financiación de los déficit. En efecto, tanto el ajuste como la recuperación cla la economía mundial dependen de un mecanismo paulatino que ayude a financiar los déficit y que, en particular, ayucle a los paises cleficitarios a sortear el psrioclo de transición.

Uno de los primeros requisitos de un ajuste financiero paulatino es una aclecuada closis de realismo de parte de los acreedores. Los acreedores deben tener en consicleración que la economia mundial atraviesa un periodo de extraordinarias dificultades y que los problemas externos que deben enfrentar los paises en desarrollo se deben largamente a factores que escapan a su control. Deben bener en cuenta también que, descle su posición de acreedores, están ob. tenienclo no despreciables benelícios.

Cuando, durante los años 1971 y 1975 , se clispuso de importantes volúmenes de recursos financieros para atender los déficit del momento, tanto acreedores como deudores actuaron partiendo del supuesto de que la recesión en los países inclustriales habria de superarse con los mecanismos y en los términos convencionales $y$ tradicionales.

$\mathrm{Si}$, como parece ser el caso, la recesión se prolongara mucho más de lo previsto, los acreedores deberán tener especialmente on cuenta lo siguiente: cuando un pais ya endeudado se enfrenta a una caida pronunciada de sus exportaciones, un buen manejo de las políticas internas puede no ser suficiente para resolver sus problemas de pagos. Otro elemento fundamental es una actitud de cooperación por parte de sus acreedores. 
Pero no basta con un adecuado volumen de créditos. Se necesita además que los mismos sean extendidos en términos azonables. Plazos breves y estrictos pueden justificarse cuando los problemas financieros se refieren a casos aislados o son atribuibles a causns puramente nacionales. Pero seria un error caer en la tentación te pensar que la necesaria liquidez de mediano plazo puede tan sólo suministrarse a través de créclitos de corto plazo. Es por ello que deberán realizarse grancles esfuerzos para que los vencimientos se pueflan extender tanto como sea posibie a fin de dar asi un margen de tiempo al ajuste interno de los paises.

Hay, además, otro mzonamiento que vale la pena enfatizal.

No debieral esperarse que las fuentes privatlas tomen a su cargo todo el riesgo de financiamiento de la economía mundia! en este periodo clifícil. De ahí el papel relevante que cleben tener en estos momentos las instituciones multilatcrales. En efecto, sólo ellis pueden proporcionar créditos oficiales en condiciones y a plazos que alivien verdaderamente las situaciones presentes, y que faciliten en es. ta forma una transición más adlecuada $y$ menos penosa en términos sociales y económicos.

Un flujo mayor de los recursos provenientes cle las instituciones multilaterales (FMI, BID, BIRF, entre otros) tenclía, además, el beneficio de fortalecer la confianza de los acreedores privados frente a los riesgos que han venido asumiendo recientemente.

Entendamos bien. Cuando subrayo la necesidad de un mayor llujo de fondos como condición fundamental para sortear las dificultades del momento, no estoy, por supuesto, sugiriendo eximir a los países cleudores de su cuota de responsabiliclad en los procesos cle ajuste. Sin embargo, no es menos evidente que los requisitos del ajuste deben ser realistas $y$ analizar'se en el contexto de las condiciones sociales y políticas de cada país.

Los ajustes -especialmente los del orden de magnitud desconocida que han planteado las recientes crisis de balance de pagos de algunos países- requieren sacrificios del ritmo de crecimiento, pero elio incile negativamente sobre los niveles de empleo e ingresos, los que, por lo general, son insatisfactorios en muchos de nucstros países. Además, las medicias tradicionales tienden a colocar unat proporción excesiva del costo del ajuste sobre los grupos sociales más desamparados. Por esta razón, la capaciclad de un país en desarrollo para realizar ajustes estí siempre condicionada por su situación relativa de pobreza y de subdesarrollo.

Sin desconocer que puecle haber casos en los que hay espacio para ajustes adicionales a los realizaclos, se necesita tener en cuenta que el rigor de los programas de estabilización debe ser attemperado por las realidades políticas y por las exigencias de la justicia social. 


\section{En conclusión:}

No cabe plantearse el problema cle pagos de algunos paises de la región en términos de una posible bancarrola. Ello no se corresponde ni con la historia de la región, ni con la madurez de las politicas internas puesta de manifiesto en los últimos años parat reaccionar frente a las soluciones, ni con la propia clinámica de la evolución económica reciente de América Latina.

Existe, sí, un problema de pagos, que podrá continuar para algunos países de la región durante los años inmediatos, pero el mismo se inserta en in cuadro de causas y de posibles söluciones que sobrepasan largamente la capacilad de estos países para resolverios aislaclamente.

Hay en todo caso una triple responsabilidad en el problema. Está, en primer término, la del mundo en su conjunto $y$, en particular, de los paises industriales que deben procurar el ajuste internacional, la recuperación de sus economias, y la solución a viejos y vitales problemas del orden económico internacional, cuya superación es hoy más necesaria que nunca.

Está, en segundo lugar, ta responsabilidad de los paises latinoamericanos mismos que, enfrentados cada uno a circunstancias particulares, deben continuar realizando un manejo serio y responsable de sus economíts, efectuando los ajustes internos, acelerando su apertura externa, pero revisando también sus propios estilos de defarrollo para extraer de las lecciones pasadas las guias para un futuro desarrollo mucho más equilibrado y sostenido.

$X^{\prime}$ está, por último, la responsabilidad de la comunidad financiera internacional, tanto pública como privada. La primera, para alentar la cooperación incernacional en la búsqueda de mayores volúmenes cle crédito internacional en mejores condiciones, a través de instituciones multilaterales. La segunda, para observar con criterios renovados una situación particular donde se necesita una gran dosis de coraje, realismo y, sobre todo, comprensión acerca de la verdadera naturaleza y la enorme complejidad de los problemas que enfren. tamos. 